\title{
Non-QCD contributions to top-pair production near threshold*
}

\section{Martin Beneke}

Physik Department T31, James-Franck-Straße 1, Technische Universität München, 85748 Garching, Germany

\section{Andreas Maier}

IPPP, Department of Physics, University of Durham, DH1 3LE, United Kingdom

\section{Jan Piclum}

Albert Einstein Center for Fundamental Physics, Institute for Theoretical Physics, Sidlerstrasse 5, CH-3012 Bern, Switzerland

\section{Thomas Rauh ${ }^{\dagger}$}

Physik Department T31, James-Franck-Straße 1, Technische Universität München, 85748 Garching, Germany

E-mail: thomas.rauh@mytum.de

\begin{abstract}
The threshold scan of top pair production at a future lepton collider allows to determine several Standard Model parameters with very high precision. The recent completion of the third-order QCD corrections to the inclusive top-pair production cross section demonstrated that strong dynamics are under control. We investigate effects from P-wave production and Higgs contributions at third order and from QED and the nonresonant production of the physical $W^{+} W^{-} b \bar{b}$ final state at first order. We discuss the sensitivity of the cross section to the top mass, width and Yukawa coupling as well as to the strong coupling.
\end{abstract}

The European Physical Society Conference on High Energy Physics

22-29 July 2015

Vienna, Austria

\footnotetext{
*TUM-HEP-1024/15

† Speaker.
} 


\section{Introduction}

The top-quark mass is an important parameter for many observables in the Standard Model and beyond, including the stability of the vacuum, due to the often large radiative corrections involving virtual top quarks. Currently, the highest precision is achieved in the direct reconstruction of (anti-) top quarks from their decay products at the Tevatron and LHC with a total uncertainty of $\pm 0.76 \mathrm{GeV}$ [1]. However, this value is plagued by the lack of understanding of the precise relationship between the measured Monte Carlo mass and a "proper" mass definition from the theory point of view, which could add an additional uncertainty of the order of $1 \mathrm{GeV}$. This can be circumvented by determinations of the top-quark mass from the measurement of the total top pair production cross section in hadron collisions [2, 3] or indirectly, from flavour and electroweak precision observables [4]. The drawback is an increased uncertainty at the level of several GeV. A measurement of the top quark mass with an uncertainty substantially below $\pm 1 \mathrm{GeV}$ can be achieved by performing a threshold scan at a future lepton collider, which consists of the measurement of the total inclusive top pair production cross section for about ten center-of-mass energies near the production threshold $\sqrt{s} \sim 2 m_{t}$ [5]-7]. By comparison of the shape of the cross section with the theory prediction the top mass can be measured directly in a well-defined short-distance mass scheme and with very high accuracy. Furthermore the top width can be determined precisely and modifications of the top Yukawa coupling through new physics effects could possibly be detected. For this program it is crucial that the level of accuracy provided by a lepton collider is matched on the theory side. The recent completion of the QCD contributions up to NNNLO precision [8] showed that the theory uncertainty is now greatly reduced with respect to the NNLO predictions [9] and at the level of just $\pm 3 \%$. Thus non-QCD effects, which can affect the cross section by up to $10 \%$ [10], have now become the focus of further theoretical efforts. In the following we give a very brief outline of the special dynamics near the production threshold, then discuss various non-QCD effects and present numerical results for the cross section and its sensitivity to different input parameters.

Threshold dynamics. In the vicinity of the top-pair production threshold $\sqrt{s} \sim 2 m_{t}$ the tops are nonrelativistic with a small velocity of the order of the strong coupling constant $v \sim \alpha_{s}$. Thus the top mass $m_{t}$, momentum $m_{t} v$ and energy $m_{t} v^{2}$ are vastly different and set the relevant scales, denoted as the hard, soft and ultrasoft scale. In addition terms scaling like $\left(\alpha_{s} / v\right)^{n}$ appear which are not suppressed in the nonrelativistic counting and indicate the breakdown of conventional perturbation theory. Hence these so-called Coulomb singularities have to be resummed to all orders. This can be achieved in the effective field theory of potential non-relativistic QCD (PNRQCD), which is obtained by subsequently integrating out the hard and soft scale. A distinguishing aspect of PNRQCD is that the LO Lagrangian does not describe free fields, but nonrelativistic top fields which are interacting through an instantaneous colour Coulomb potential. Consequently the leading order Coulomb interaction is treated nonperturbatively while higher order corrections can be obtained systematically by expanding in $\alpha_{s}$ and $v$ around the resummed solution. For more details on the EFT framework we refer to the literature [11], where everything required for the NNNLO cross section is described. The cross section, normalized as usual to the muon pair production cross section, can be expressed using the optical theorem as

$$
R(s) \equiv \frac{\sigma\left(e^{+} e^{-} \rightarrow \gamma^{*}, Z^{*} \rightarrow t \bar{t} X\right)}{\sigma_{0}\left(e^{+} e^{-} \rightarrow \mu^{+} \mu^{-}\right)}=12 \pi f(s) \operatorname{Im}\left[\Pi^{(v)}(s)\right]
$$



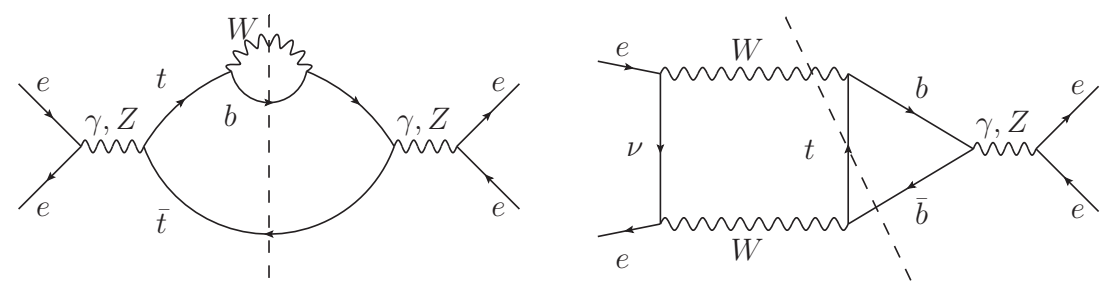

Figure 1: Sample diagrams for the nonresonant production of the $W^{+} W^{-} b \bar{b}$ final state.

where $f(s)=e_{t}^{2}+\ldots$ is a prefactor depending on the top couplings to photons and $Z$ bosons and kinematic variables. The vector polarization function $\Pi^{(v)}(s)$ has the form

$$
\Pi^{(v)}(s)=\frac{3}{2 m_{t}^{2}} c_{v}\left[c_{v}-\frac{E}{m_{t}}\left(c_{v}+\frac{d_{v}}{3}\right)\right] G(E)+\ldots,
$$

where $E=\sqrt{s}-2 m_{t}$ is the energy of the top pair, $c_{v}, d_{v}$ are hard matching coefficients for the external vector current, and the Green function $G(E)$ describes the propagation of the top pair within PNRQCD, subject to interactions from various potentials and the exchange of ultrasoft gluons. The imaginary part of the vector polarization function is known to third order in the reorganized and resummed expansion in $\alpha_{s}$ and $v$, see Figure 1 of [8]].

\section{Non-QCD and P-wave contribution}

In the following we discuss further effects not contained in the QCD vector polarization function, which are parametrically or numerically of similar size as the remaining $\pm 3 \%$ theoretical uncertainty on the contribution from $\Pi^{(v)}(s)$.

$P$-wave contribution. In addition to the dominant contribution from the vector current as described above, the top pair can also be produced through an axial-vector current from the exchange of a $s$-channel $\mathrm{Z}$ boson. This yields top pairs in a P-wave state which are suppressed by a factor $v^{2}$ with respect to the leading S-wave production and thus constitutes a NNLO effect. The full contribution up to NNNLO has been computed and discussed in [12]. This correction is only of the order of $1 \%$ relative to the third-order S-wave QCD result [8], and is included in what is referred to as the QCD prediction below.

Higgs effects. In the following we consider only Higgs effects that come from the top Yukawa coupling. Contributions involving the coupling to gauge bosons will be regarded as general electroweak effects and treated separately. The former manifest themselves as corrections to the hard matching coefficient $c_{v}$ of the external vector current and in a local contribution to the $t \bar{t}$ potential. ${ }^{1}$ The pure Higgs contribution to $c_{v}$ has been computed in [15-17] and mixed Higgs and QCD corrections in [17]. The insertion of the Higgs potential into the Green function was calculated recently in [10], such that the full NNNLO Higgs correction to the cross section is now known.

\footnotetext{
${ }^{1}$ Earlier work included the potential induced by Higgs exchange in the form of a Yukawa potential [13, 14]. Consistency with the implementation of the matching coefficients requires that the Yukawa potential is approximated by a local potential when the Higgs mass is much larger than the typical potential momentum exchange, and treated as a perturbation.
} 

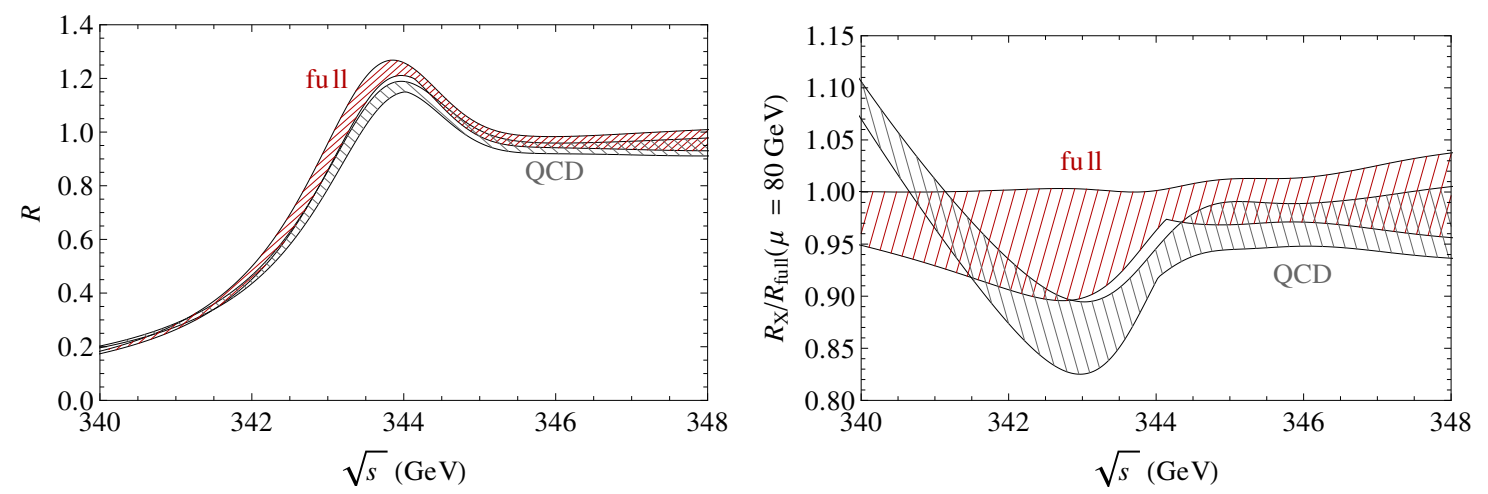

Figure 2: Overall effect of non-QCD corrections on the cross section. The uncertainty band is spanned by variation of the renormalization scale $\mu \in[50 \mathrm{GeV}, 350 \mathrm{GeV}]$. In the right plot the cross section is normalized to the full one at the central scale $\mu=80 \mathrm{GeV}$. Figures from [10].

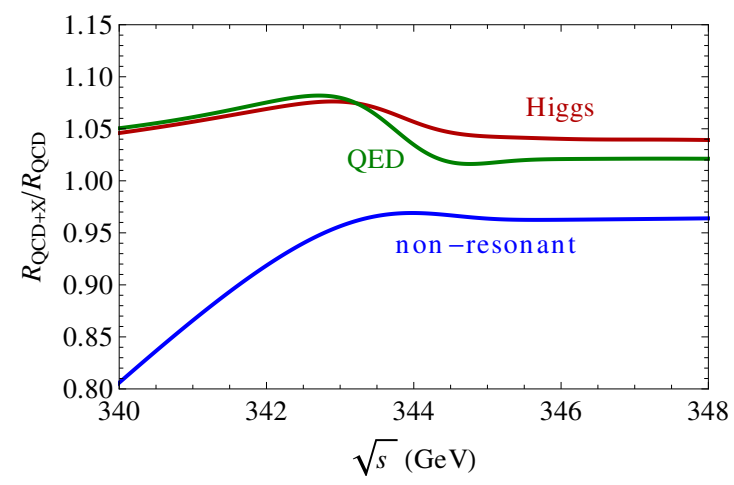

Figure 3: Relative size of the different non-QCD corrections to the top-pair production cross section with respect to the pure QCD result at $\mu=80 \mathrm{GeV}$. Figure from [10].

Nonresonant effects. Since the cross section near threshold is also sensitive to the small ultrasoft scale and the top width is of the same order, the narrow-width approximation cannot be used here to factorize the production and decay of the top pair. This implies that, assuming $V_{t b}=1$, the physical final state is $W^{+} W^{-} b \bar{b}$. It is dominantly produced through a resonant top pair, where the replacement $E \rightarrow E+i \Gamma_{t}$ accounts for the effects of top instability [18]. At higher orders in the nonrelativistic counting the $W^{+} W^{-} b \bar{b}$ final state can however also be produced with just one or no resonant tops. Two sample diagrams at NLO without an on-shell top (left) or anti-top (right) are shown in Figure 11. Only the sum of both processes constitutes a physical quantity as is also apparent from singularities that appear in both parts at NNLO and only cancel in the sum. In a systematic way the two contributions can be organized within Unstable Particle Effective Theory [19, 20]. The nonresonant NLO effects have been calculated in [21] and have been included in [10]. At NNLO only partial results are available [22-24], which we have not considered yet.

QED effects. The leading electroweak correction is the QED Coulomb potential at NLO. Its contribution can be inferred from the results available from the QCD calculation and has been included in [10]. Further electroweak effects at NNLO [15, 16, 25, 26] and even at NNNLO [17, 27] are known, but have not been included yet, since the full NNLO nonresonant correction is not 

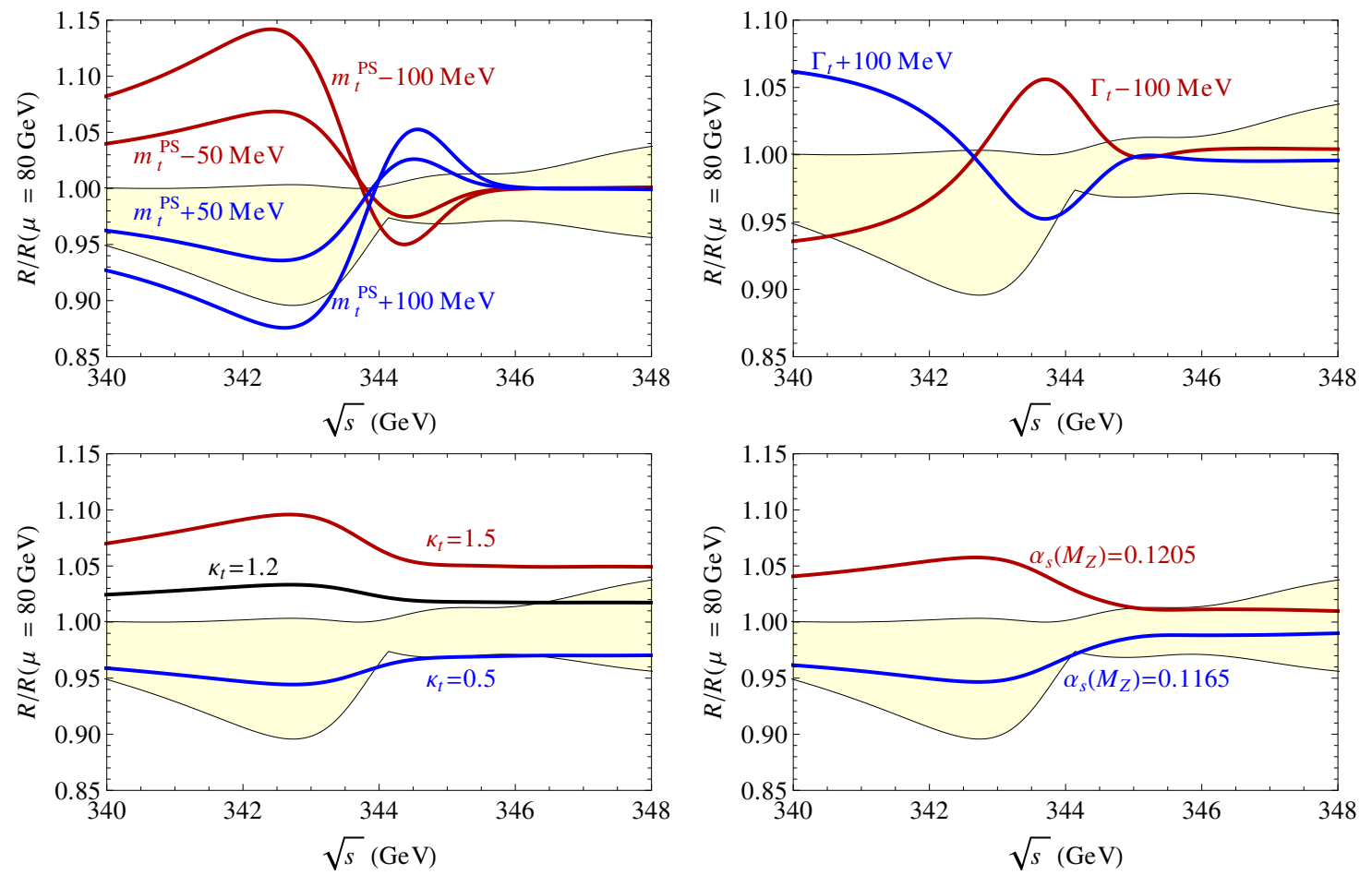

Figure 4: The relative change of the cross section under variations of the top mass, width and Yukawa coupling as well as the strong coupling constant is shown in comparison to the uncertainty band obtained by scale variation. Figures in the second row from [10]; those in the first row are similar to the ones shown in [8], except that now the cross section includes the P-wave and non-QCD contributions discussed in [10] and this proceeding.

available yet and thus no complete description of EW effects at this order is possible at the moment.

\section{Phenomenology}

We compare the non-QCD effects described above to the pure QCD cross section. The latter is given by the results of [8] to which we add the small P-wave contribution [12]. The net effect is shown in Figure 2, where the uncertainty bands for the pure QCD and the full result are displayed (see [10] for the adopted parameter values). We observe that the non-QCD contributions change the cross section by up to about $10 \%$ and particularly affect the shape of the cross section at and below threshold. The shift is larger than the QCD uncertainty estimate, thus it is very important to include these contributions. Based on the shift in the peak position we estimate that the effect on the measurement of the top mass is approximately $50 \mathrm{MeV}$, which is the expected total uncertainty. The separate corrections relative to the QCD prediction are shown in Figure 3. The Higgs and QED contributions both increase the cross section by $4-8 \%$ and $2-8 \%$, respectively, since they provide an additional attraction between the top pair. Furthermore they shift the peak slightly towards smaller center-of-mass energies. ${ }^{2}$ On the other hand the nonresonant contribution is negative, in-

\footnotetext{
${ }^{2}$ The peak arises from the smeared out toponium resonances, whose binding energy is increased by the additional attractive potentials.
} 


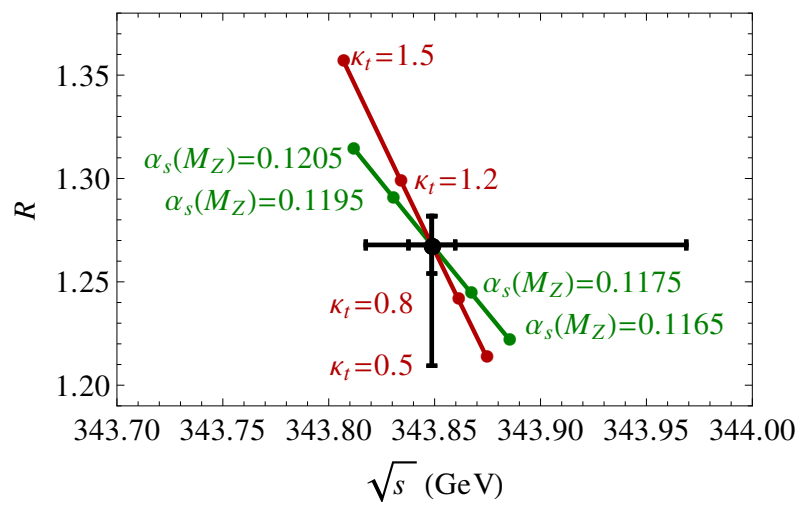

Figure 5: Changes in peak height and position due to variation of the Yukawa coupling (red line) and the strong coupling (green line). The black error bars denote the $\alpha_{s}$ and combined scale and $\alpha_{s}$ uncertainty for $y_{t}=y_{t}^{\mathrm{SM}}\left(\kappa_{t}=1\right)$ and $\alpha_{s}\left(M_{Z}\right)=0.1185$. Figure from [10].

sensitive to the special dynamics near threshold, and roughly energy-independent at NLO. This implies that the relative correction can reach up to $20 \%$ below threshold, where the cross section becomes small.

To get an idea of the physics potential of a top threshold scan at a future lepton collider we discuss the dependence of the cross section on the input parameters and compare it to the theory uncertainty. Relative to the full result at $\mu=80 \mathrm{GeV}$ this is shown in Figure 4 A change in the top mass mainly manifests itself in a horizontal shift of the cross section by twice that amount. An increase/decrease of the top width changes the degree to which the toponium resonances are smeared out and thus makes the peak in the cross section less/more pronounced. The parameter $\kappa_{t}$ parametrizes possible new physics effects in the relation between the top Yukawa coupling and mass $y_{t}=\sqrt{2} \kappa_{t} m_{t} / v$, where $\kappa_{t}=1$ corresponds to the Standard Model. Variation of $\kappa_{t}$ as well as the strong coupling mainly changes the normalization of the cross section. Due to the similar effect on the cross section the sensitivity to the individual parameters $\kappa_{t}, \alpha_{s}$ in a threshold scan is reduced if both are extracted in a simultaneous fit. For the peak position and height these dependences are illustrated in Figure 5. Given the small error of the strong coupling constant it can also be used as an external input, in which case the added uncertainty relative to the scale variation is small.

A rough estimate of the theory uncertainty in measurements of these parameters can be obtained by determining the parameter shifts for which the change of cross section lies outside of the uncertainty band in Figure 4 . This however underestimates the sensitivity, since in the threshold scan the cross section is measured for multiple points and the theory uncertainty is (at least to some degree) correlated. A reliable estimate on the sensitivity can thus only be obtained by an experimental study using the full theory result. Unfortunately this is not available yet, but existing studies [5]-7] with less complete theory input suggest that the experimental uncertainties are about one half of the present theoretical ones for the top width and mass, specifically of the order of $20 \mathrm{MeV}$ for the mass and $20-30 \mathrm{MeV}$ for the width, respectively, and 0.001 for the strong coupling and $5-15 \%$ for the top Yukawa coupling.

Acknowledgements. This work was supported by the Gottfried Wilhelm Leibniz programme of the Deutsche Forschungsgemeinschaft (DFG) and the DFG cluster of excellence "Origin and 
Structure of the Universe".

\section{References}

[1] ATLAS and CDF and CMS and D0 Collaborations, arXiv:1403.4427 [hep-ex].

[2] ATLAS Collaboration, Eur. Phys. J. C 74 (2014) 10, 3109 [arXiv:1406.5375 [hep-ex]].

[3] CMS Collaboration, Phys. Lett. B 728 (2014) 496, Corrigendum-ibid. B 728 (2014) 526 [arXiv:1307.1907 [hep-ex]].

[4] G. F. Giudice, P. Paradisi and A. Strumia, arXiv:1508.05332 [hep-ph],

[5] M. Martinez and R. Miquel, Eur. Phys. J. C 27 (2003) 49 hep-ph/0207315].

[6] K. Seidel, F. Simon, M. Tesař and S. Poss, Eur. Phys. J. C 73 (2013) 8, 2530 [arXiv:1303.3758 [hep-ex]].

[7] T. Horiguchi, A. Ishikawa, T. Suehara, K. Fujii, Y. Sumino, Y. Kiyo and H. Yamamoto, arXiv:1310.0563 [hep-ex].

[8] M. Beneke, Y. Kiyo, P. Marquard, A. Penin, J. Piclum and M. Steinhauser, arXiv:1506.06864 [hep-ph], accepted for publication in Phys. Rev. Lett.

[9] A. H. Hoang et al., Eur. Phys. J. direct C 3 (2000) 1 hep-ph/0001286].

[10] M. Beneke, A. Maier, J. Piclum and T. Rauh, Nucl. Phys. B 899 (2015) 180 [arXiv:1506.06865 [hep-ph]].

[11] M. Beneke, Y. Kiyo and K. Schuller, arXiv:1312.4791 [hep-ph].

[12] M. Beneke, J. Piclum and T. Rauh, Nucl. Phys. B 880 (2014) 414 arXiv:1312.4792 [hep-ph].

[13] M. J. Strassler and M. E. Peskin, Phys. Rev. D43 (1991) 1500.

[14] R. Harlander, M. Jeżabek and J. H. Kühn, Acta Phys. Polon. B27 (1996) 1781 hhep-ph/9506292].

[15] B. Grzadkowski, J. H. Kühn, P. Krawczyk and R. G. Stuart, Nucl. Phys. B 281 (1987) 18.

[16] R. J. Guth and J. H. Kühn, Nucl. Phys. B 368 (1992) 38 .

[17] D. Eiras and M. Steinhauser, Nucl. Phys. B 757 (2006) 197 hep-ph/0605227].

[18] V. S. Fadin and V. A. Khoze, JETP Lett. 46 (1987) 525 Pisma Zh. Eksp. Teor. Fiz. 46 (1987) 417].

[19] M. Beneke, A. P. Chapovsky, A. Signer and G. Zanderighi, Phys. Rev. Lett. 93 (2004) 011602 hep-ph/0312331.

[20] M. Beneke, A. P. Chapovsky, A. Signer and G. Zanderighi, Nucl. Phys. B 686 (2004) 205 hep-ph/0401002].

[21] M. Beneke, B. Jantzen and P. Ruiz-Femenía, Nucl. Phys. B 840 (2010) 186 [arXiv:1004.2188 [hep-ph]].

[22] A. A. Penin and J. H. Piclum, JHEP 1201 (2012) 034 arXiv:1110.1970 [hep-ph]].

[23] B. Jantzen and P. Ruiz-Femenía, Phys. Rev. D 88 (2013) 5, 054011 arXiv:1307.4337 [hep-ph].

[24] P. Ruiz-Femenía, Phys. Rev. D 89 (2014) 9, 097501 arXiv:1402.1123 [hep-ph]].

[25] A. H. Hoang and C. J. Reißer, Phys. Rev. D 71 (2005) 074022 hep-ph/0412258].

[26] A. H. Hoang and C. J. Reißer, Phys. Rev. D 74 (2006) 034002 hep-ph/0604104].

[27] Y. Kiyo, D. Seidel and M. Steinhauser, HHEP 0901 (2009) 038 arXiv:0810.1597 [hep-ph]. 